\title{
A new look at acid catalyzed deacetylation of carbohydrates : A regioselective synthesis and reactivity of 2-O-acetyl aryl glycopyranosides
}

\section{Stepanova, Elena V.}

2018-03-22

Stepanova , E V , Nagornaya, M O, Filimonov, V D , Valiev, R R, Belyanin , M L , Drozdova , A K \& Cherepanov , V N 2018 , ' A new look at acid catalyzed deacetylation of carbohydrates: A regioselective synthesis and reactivity of 2-O-acetyl aryl glycopyranosides ' , Carbohydrate Research , vol. 458 , pp. 60-66 . https://doi.org/10.1016/j.carres.2018.02.003

http://hdl.handle.net/10138/311656

https://doi.org/10.1016/j.carres.2018.02.003

cc_by_nc_nd

acceptedVersion

Downloaded from Helda, University of Helsinki institutional repository.

This is an electronic reprint of the original article.

This reprint may differ from the original in pagination and typographic detail.

Please cite the original version. 


\section{Accepted Manuscript}

A new look at acid catalyzed deacetylation of carbohydrates: A regioselective synthesis and reactivity of 2-O-acetyl aryl glycopyranosides

Elena V. Stepanova, Marina O. Nagornaya, Victor D. Filimonov, Rashid R. Valiev, Maxim L. Belyanin, Anna K. Drozdova, Victor N. Cherepanov

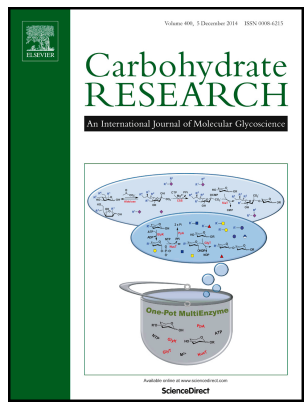

PII:

S0008-6215(17)30915-1

DOI:

10.1016/j.carres.2018.02.003

Reference: CAR 7519

To appear in: Carbohydrate Research

Received Date: 16 December 2017

Revised Date: 28 January 2018

Accepted Date: 4 February 2018

Please cite this article as: E.V. Stepanova, M.O. Nagornaya, V.D. Filimonov, R.R. Valiev, M.L. Belyanin, A.K. Drozdova, V.N. Cherepanov, A new look at acid catalyzed deacetylation of carbohydrates: A regioselective synthesis and reactivity of 2-O-acetyl aryl glycopyranosides, Carbohydrate Research (2018), doi: 10.1016/j.carres.2018.02.003.

This is a PDF file of an unedited manuscript that has been accepted for publication. As a service to our customers we are providing this early version of the manuscript. The manuscript will undergo copyediting, typesetting, and review of the resulting proof before it is published in its final form. Please note that during the production process errors may be discovered which could affect the content, and all legal disclaimers that apply to the journal pertain. 


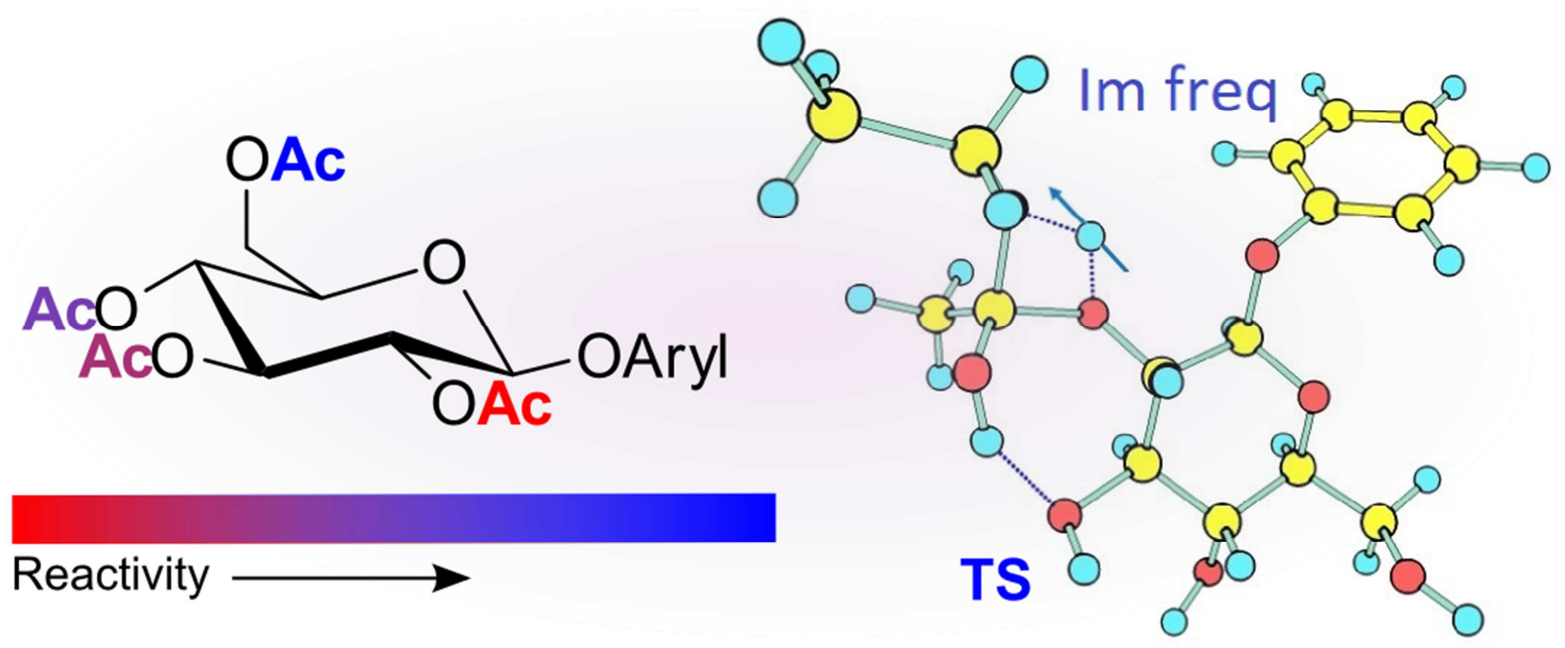




\title{
A new look at acid catalyzed deacetylation of carbohydrates: a
}

\section{regioselective synthesis and reactivity of 2-O-acetyl aryl glycopyranosides}

\author{
Elena V. Stepanova, ${ }^{\mathrm{a} 1}$ Marina O. Nagornaya, ${ }^{\mathrm{a}}$ Victor D. Filimonov, ${ }^{\mathrm{a}}$ Rashid R. Valiev, ${ }^{\mathrm{b}, \mathrm{c}}$ \\ Maxim L. Belyanin, ${ }^{a}$ Anna K. Drozdova, ${ }^{b}$ Victor N. Cherepanov ${ }^{b}$
}

${ }^{a}$ Department of Biotechnology and Organic Chemistry, National Research Tomsk Polytechnic University, 30 Lenin Avenue, Tomsk 634050, Russia

${ }^{\mathrm{b}}$ Tomsk State University, Lenin Avenue 36, Tomsk 634050, Russia

${ }^{c}$ University of Helsinki, Department of Chemistry, P.O. Box 55 (A.I. Virtanens plats 1), FIN00014 University of Helsinki, Finland

\begin{abstract}
In the present work we report that acetyl groups of per - acetylated aryl glycosides have different reactivity during the acidic deacetylation using $\mathrm{HCl} / \mathrm{EtOH}$ in $\mathrm{CHCl}_{3}$, which leads to preferential deacetylation at O-3, O-4 and O-6. Thereby, the one-step preparation of 2-O-acetyl aryl glycosides with simple aglycon was accomplished for the first time. It was proved that the found reagent is to be general and unique for the preparation of series of 2-O-acetyl aryl glycosides. We have determined the influence of both carbohydrate moiety and the aglycon on the selectivity of deacetylation reaction by kinetic experiments. Using DFT/B3LYP/6-31G(d,p) and semi-empirical AM1 methods we have found that the highest activation barrier is for 2-Oacetyl group. This completely explains the least reactivity of 2-O-acetyl group.

\footnotetext{
${ }^{1}$ Corresponding author at present address: Department of Biotechnology and Organic Chemistry, National Research Tomsk Polytechnic University, 30 Lenin Avenue, Tomsk, 634050, Russia. Tel.: 7 (3822) 5638 61; fax: +7 (3822) 563637.

E-mail addresses: eline_m@mail.ru, glycoside.m@gmail.com (E.V. Stepanova).
} 
Key words: aryl glycosides, regioselective deacetylation, reactivity, partially acylated carbohydrates, transition states modeling 
Highlights:

- Different reactivity of acetyl groups of glycosides in acid-catalyzed deacetylation

- Regioselective preparation of 2-O-acetyl aryl glycosides was accomplished

- The influence of carbohydrate structure on regioselectivity of deacetylation

- Different reactivity of Ac groups is explained by activation barriers 


\section{Introduction}

Aryl glycosides are one of the most common plant secondary metabolites and are of interest as naturally occurring bioactive substances. Aryl glycosides play an important role in plant development and interaction with other organisms, namely protection of plants from microbes, insects and herbivores [1]. A large number of studies have been carried out to establish antiparasitic, antitumor, antiviral activity of arylglycosides [2,3].

Partially acetylated aryl glycosides are widely found in nature. Particularly, many examples of 2-O-acetyl arylglycosides can be found [4, 5]. Several 2-O-acetyl arylglycosies are known as chemotaxonomic markers for the plants of Salicaceae family such as 2-acetylsalicin [6] and 2-acetylsalicortin [7]. Partially acetylated aryl glycosides are also interesting for pharmacology as they have different bioactivity comparing to non-acetylated compounds with the same skeleton $[8,9]$. Synthesis of these compounds was not accomplished to date due to the complex procedure of the selective introduction of a single acetyl group into the molecule of glycoside and the feasibility of acetyl group to migrate $[10,11,12,13]$. Only few examples of synthesis of 2-O-acetyl aryl glycosides with simple aglycon are published requiring many steps [14].

Generally, acetyl groups are widely used as protective in carbohydrate chemistry [15]. In some syntheses 2-O-acyl protecting group in carbohydrate moiety might be strongly required for the selective trans - glycosylation as a participating group [16]. Thus, 2-O-acetyl gluco- and galactopyranosyl building blocks can find application in oligosaccharide synthesis. To remove acetyl groups from carbohydrate moiety base- catalyzed methods such as Zemplen [17] or similar $[18,19,20]$ procedures are commonly used. Acidic catalysis is applied only in the case of selective removal of acetyl group in the presence of benzoyl [21] or other ester groups [22, 23, 24, 25]. However, acidic catalysis is unfavorable in carbohydrate chemistry due to the low stability of glycosidic linkage in acidic medium [26] and thus has less attention in syntheses. 
Nevertheless, in the present work we show that acidic - catalyzed deasetylation can find wider applications in carbohydrate synthesis due to the different reactivity of acetyl groups.

In our previous work, we demonstrated that the treatment of per - acetylated glycosides bearing bulky ortho - substituted aglycon with $\mathrm{HCl}$ in $\mathrm{EtOH}$ and $\mathrm{CHCl}_{3}$ reagent does not break the glycosidic bond and selectively removes three acetyl groups. As a result, the 2-O-acetyl glycosides were prepared with moderate to high yields [27]. In the present research we report that bulky ortho - substituted phenols are not obligatory to achieve selectivity and any aryl aglycon can be present for the preparation of 2-O-acetyl glycosides. We study the influence of both carbohydrate moiety and the aglycon on regioselectivity of deacetylation in the described conditions $\left(\mathrm{HCl}\right.$ in $\mathrm{EtOH}$ and $\left.\mathrm{CHCl}_{3}\right)$. We also elucidate the nature of this phenomenon using experimental and theoretical methods.

\section{Materials and methods}

\subsection{General}

Silicagel MN Kieselgel $600.04-0.063 \mathrm{~mm}$ was used for column chromatography. Commercially available solvents were used after drying, distillation and were kept under molecular sieves $4 \square$. HPLC analysis was carried out on an Agilent Compact LC with a $150 \times 4.6$ mm Eclipse Plus C-18 (5 $\mu \mathrm{m})$ column, eluted with a gradient of $\mathrm{H}_{2} \mathrm{O}-\mathrm{CH}_{3} \mathrm{CN}$ containing $0.1 \%$ trifluoroacetic acid from $0 \%$ to $100 \% \mathrm{CH}_{3} \mathrm{CN}$ in $20 \mathrm{~min}$ and a flowrate of $1 \mathrm{~mL} / \mathrm{min}$. Probe volume was $20 \mu \mathrm{L}$. UV detection was performed at $220 \mathrm{~nm}$. The preparation of glycosides $\mathbf{1 - 9}$ was carried out according to the known procedure from 1,2,3,4,6-tetra-O-acetyl- $\beta$-Dglucopyranose or 1,2,3,4,6-tetra-O-acetyl- $\beta$-D-galactopyranose using $\mathrm{BF}_{3} \cdot \mathrm{Et}_{2} \mathrm{O}$ and $\mathrm{Et}_{3} \mathrm{~N}$ [28]. All physicochemical data for glycosides 1-9 was in good agreement with those published in literature.

\subsection{General deacetylation procedure}


To a solution or suspension of per-acetyl glucoside 1-9 $(1 \mathrm{mmol})$ in $\mathrm{CHCl}_{3}(1 \mathrm{ml})$ and $96 \%$

EtOH (3 ml) a 36\% solution of hydrochloric acid $(1 \mathrm{ml}, 10 \mathrm{mmol})$ was added and the complete dissolution of glycoside was observed. The reaction mixture was thermostated at $30{ }^{\circ} \mathrm{C}$ until HPLC showed the maximal peak of 2-O-acetyl glycoside (retention times are given in Table 1). The reaction mixture was treated with anion-exchange resin $\mathrm{AB}-17$ until a $\mathrm{pH}$ of 7 was reached, filtered, and concentrated under vacuum. The residue was subjected to column chromatography using $\mathrm{CHCl}_{3}-\mathrm{EtOH}$ 15:1 $\rightarrow$ 4:1 as eluents to give 2-O-acetyl glycosides 1a-8a and nonacetylated glycosides $\mathbf{1 b}-\mathbf{9 b}$. Spectral characteristics of all resulted 2-O-acetyl glucopyranosides 1a-8a are given in the Support information file 1.

\subsection{Optimization of reaction conditions}

The optimization was carried out using glycoside $\mathbf{1}$ and the following parameters (HPLC monitoring):

1. Reaction temperature. Temperature less than $30{ }^{\circ} \mathrm{C}$ inhibits the reaction however does not influence the selectivity. Temperature higher than $30{ }^{\circ} \mathrm{C}$ both increase the reaction rate and significantly decreases selectivity.

2. Solvent composition. Varying the ethanol-chloroform ratio leads to the loss of homogeneity to form either two non-mixable phases or the crystallization of glycoside $\mathbf{1}$. Both cases lead to not comparable and unpredictable results.

3. The amount (concentration) of $\mathrm{HCl}$. We use $36 \% \mathrm{HCl}$ with the total concentration in the reaction mass of $2 \mathrm{~mol} / \mathrm{L}$. The lowering of $\mathrm{HCl}$ concentration leads to the reduction of reaction rate but no yield increase of 2-O-acetates was observed. The higher concentrations of $\mathrm{HCl}$ lead to the higher reaction rates and less selectivity.

Thus, the less reaction temperatures and lower $\mathrm{HCl}$ concentrations can be used to achieve similar reaction products but requires more time. However, higher temperatures and $\mathrm{HCl}$ concentrations are not recommended due to the loss of selectivity

\subsection{Kinetic calculations}


Rate constant was measured for the reaction of a single 2-O-acetyl group ethanolysis of glycoside 1a (Scheme 1). Calibration curve (the dependence of peak area from concentration) was prepared for glycoside $1 \mathrm{a}$ at concentrations $0.25 \mathrm{mg} / \mathrm{ml}, 0.5 \mathrm{mg} / \mathrm{ml}$ and $1 \mathrm{mg} / \mathrm{ml}$ using HPLC (Figure $1 \mathrm{~A}$ ). The $0.5 \mathrm{ml}$ ampule with screwed cap was filled with 2-O-acetyl glucoside 1a $(5 \mathrm{mg}, 16.7 \mu \mathrm{mol})$ and the mixture of $\mathrm{EtOH}(28.5 \mu \mathrm{l}), \mathrm{CHCl}_{3}(12.5 \mu \mathrm{l})$ and $\mathrm{HCl} 36 \%(4.5 \mu \mathrm{l}$, $45 \mu \mathrm{mol}$ ) with the total concentration of $\mathrm{HCl} 1 \mathrm{M}$. The ampoule was closed tightly and thermostated at $30{ }^{\circ} \mathrm{C}$. At pre-selected times the $2 \mu \mathrm{l}$ aliquot was taken, quenched with the mixture of acetonitrile - water 1:1 (200 $\mu \mathrm{l})$ and analyzed by HPLC three times and the average peak area was calculated. The concentrations of compound $\mathbf{1 a}$ in the reaction mixture were found according to the calibration curve. The graphics for the dependences of concentration of 1a (C) on time (t) (Figure $1 \mathrm{~B}$ ) and $\ln (\mathrm{C})$ on $\mathrm{t}($ Figure $1 \mathrm{C})$ were plotted. The reaction rate constant $k$ was estimated from the slope (Figure $1 \mathrm{C}$ ) assuming the reaction to be first-order in [glycoside 1a].

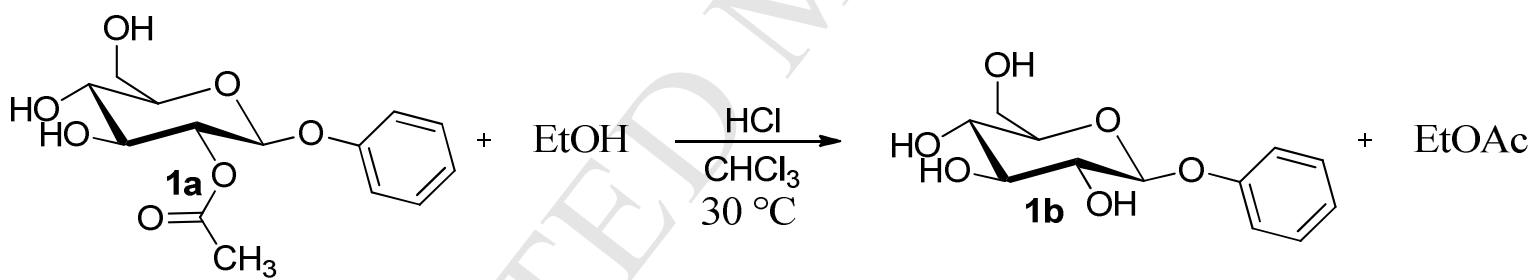

Scheme 1: The reaction of glycoside 1a ethanolysis 


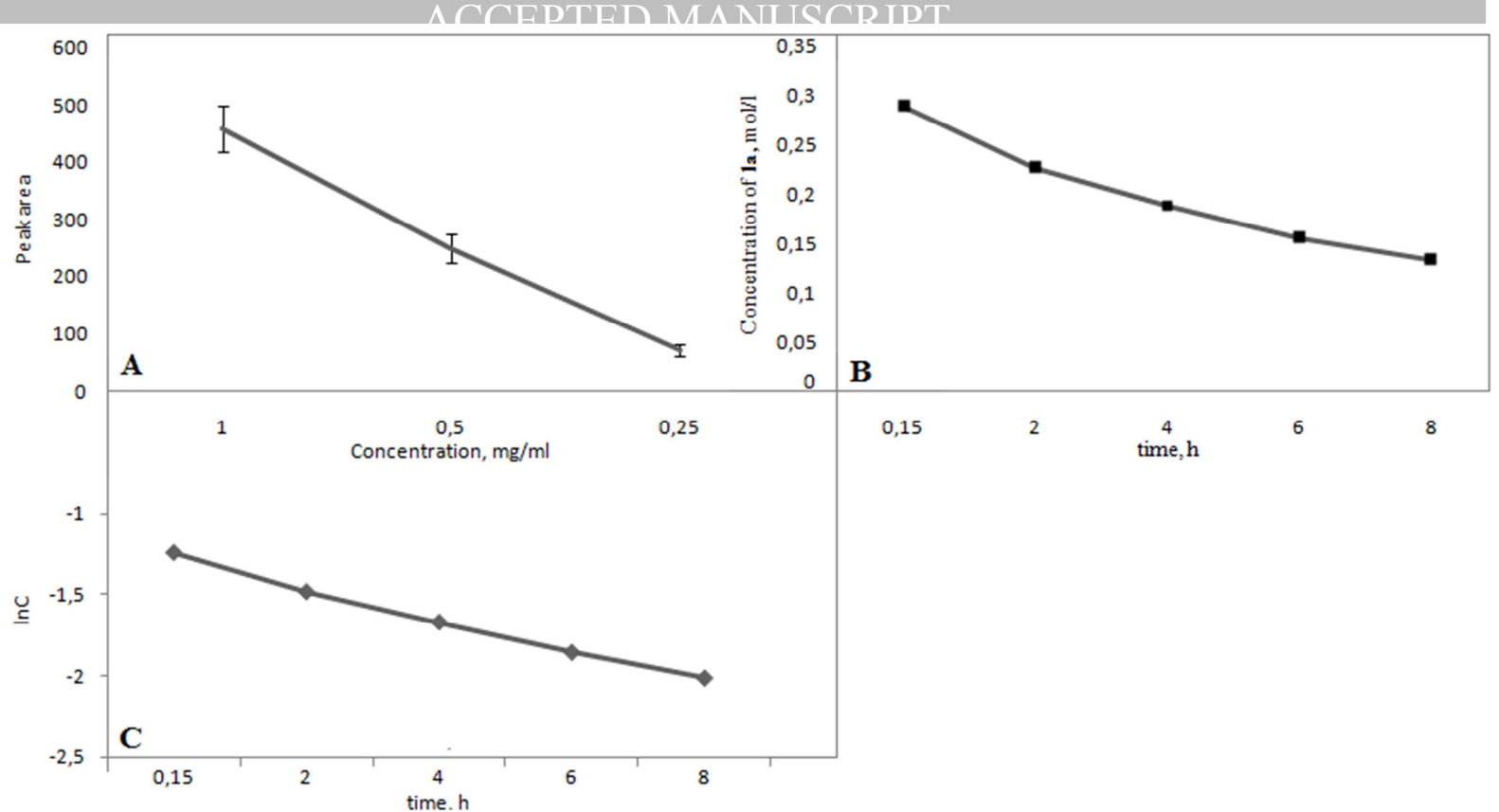

Figure 1: Kinetic measurements for the reaction of acetyl group of compound 1 ethanolysis. A): the calibration curve for glycoside 1a which shows the dependence of peak area (HPLC) from the concentration of glycoside 1a; B): the concentration profile (mol/L) of glycoside 1a during the reaction; C): linear plot for the dependence of $\ln (\mathrm{C})$ of glycoside $1 \mathrm{a}$ on time.

The described procedures were applied also for all samples 2a-8a to estimate rate constants of 2-O-acetyl group ethanolysis. The fitting of the experimental data was satisfactory for all the samples. The values of rate constants, obtained from different runs carried out under the same experimental conditions, were reproducible to within $9 \%$.

Activation energy was estimated experimentally for glycoside 1a in the same manner, when thermostating the reaction at 30,40 and $50{ }^{\circ} \mathrm{C}$ and rate constants were determined for the reactions at each temperature. Activation energy was estimated from the slope using Arrhenius graph (Figure 2, see also Table 4 in the section 3.2). 


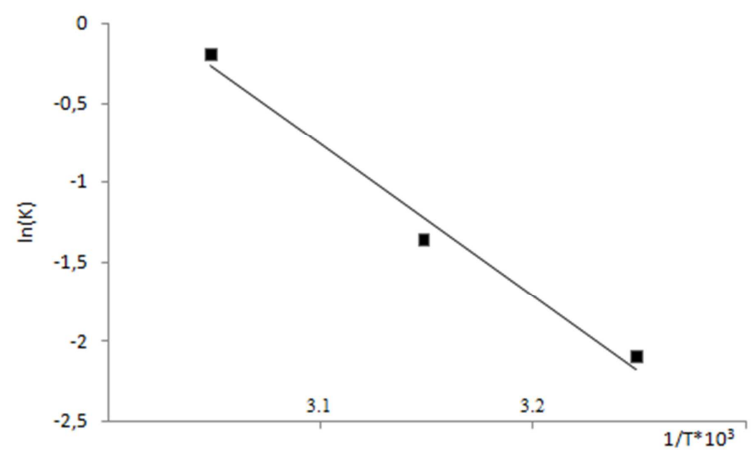

Figure 2: Arrhenius plot for the reaction of 2-O-acetyl group ethanolysis of 2-O-acetyl phenyl glucopyranoside 1a.

\subsection{Quantum chemical modeling}

The acid catalyzed transesterification reaction at low concentrations of acids is generally accepted to occur via a stepwise mechanism [29]. It includes the formation of a protonated adduct $\mathbf{I}$ and then the rate-determining step which is the nucleophilic attack with the formation of transition state (TS) [30] (Scheme 2). For the modeling and investigation of these reaction steps the quantum chemical model was built using the AM1 [31] semi-empirical method and density functional theory (DFT) [32] using the B3LYP [33] functional and 6-31G(d,p) basis set.

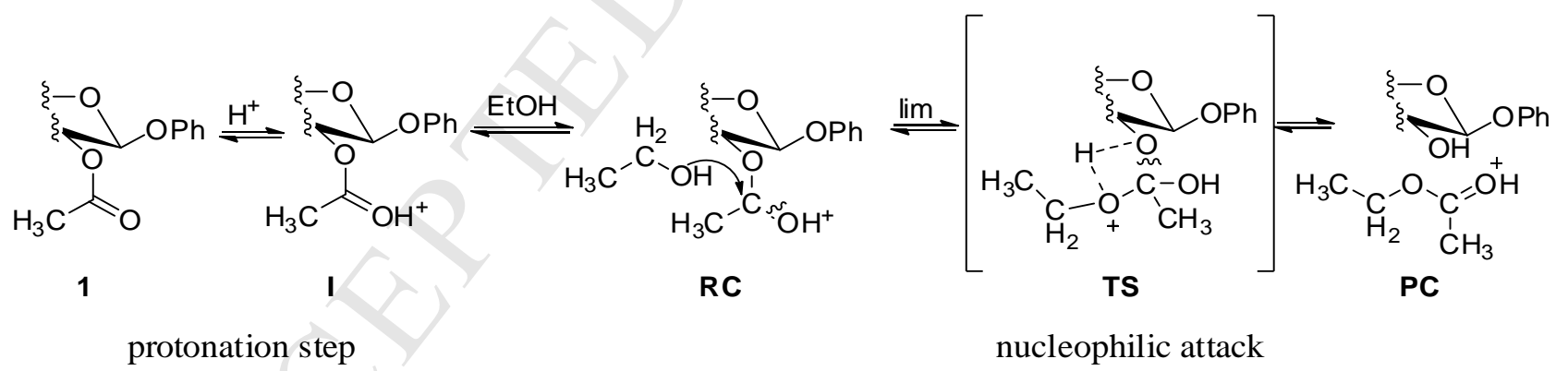

Scheme 2: Mechanism of acid-catalyzed ethanoholysis of 2-O-acetyl group for phenyl 2,3,4,6tetra-O-acetyl glucopyranoside (1).

The first step (protonation) is not rate-determining whereas the second step (nucleophilic attack) determines and characterizes the whole process and the reactivity of substrates [34]. Therefore, for the first step we calculated only Gibbs energies to characterize the reaction within the thermodynamic criteria, and for the second step we modeled the transition states, and calculated both Gibbs energies and activation barriers. 
To calculate Gibbs energies for the first (protonation) step the equilibrium geometries of ground electronic states of reagents and products were modeled at AM1 level of theory in the gas phase. The following structures of reagents were modeled: the per - acetylated glycoside $\mathbf{1}$ and the complex of proton and ethanol. The most stable complex consists of one proton and three ethanol molecules [30]. As a result of the first step of reaction the products are protonated intermediate $\mathbf{I}$ and a trimer of ethanol.

The second step (nucleophilic attack) involves the formation of reactant complex $\mathbf{R C}$ from the protonated glycoside $\mathbf{1}$ and one ethanol molecule. In TS, the tetrahedral intermediate decomposes to form a new ester (ethyl acetate) and 2-OH of glucoside (as demonstrated on Scheme 2). As a result, the product complex PC appears. The equilibrium geometries of ground electronic states for both $\mathbf{R C}$ and PC were obtained at AM1 level of theory. In order to calculate the activation barrier the geometries of $\mathbf{T S}$ were received using AM1 method.

Note that the nucleophilic attack can be the interaction between water molecule and the protonated glycoside $\mathbf{1}$ due to the water is present in the solvent composition. However, the amount of water in reaction mass is almost four times less than ethanol. We obtained the TS and activation energy for this reaction, too at AM1 level of theory.

Also, the activation energy of 2-O-acetyl phenylglucoside (1a) deacetylation was calculated at AM1 and B3LYP/6-31G(d,p) levels of theory. For the verification of using levels of theory the comparison of theoretical and experimental values of activation energy was carried out.

Cartesian coordinates of atoms for all optimized geometries are given in the Support information file 2. At present work, the thermal correction was used for all calculations at $\mathrm{T}=303$ $\mathrm{K}$ because all experimental reactions occurred at this temperature. Due to inadequate results of COSMO [35] and PCM [36] solvation models in the case of ionic compounds, we carried out all calculations for a gas phase. It should be noted, that semi-empirical AM1 method demonstrates 
good qualitative results [37] and is comparable with the density functional results [38], too. So, we chose this method for the calculation.

All calculations were carried out using GAUSSIAN software [39].

\section{Results and discussion}

\subsection{Kinetic study}

In the present work, we found that acetyl groups of per - acetylated aryl glycosides 1-9 have different reactivity in the reaction of acidic deacetylation using the following conditions: 10 M hydrochloric acid $(1 \mathrm{~mL})-96 \% \mathrm{EtOH}(3 \mathrm{~mL})-\mathrm{CHCl}_{3}(1 \mathrm{~mL})$ per $1 \mathrm{mmol}$ of tetraacetyl aryl glycoside 1-9 (Table 1). Such solvent ratio allows obtaining completely homogenous reaction mixture. We found that the main products of this reaction are 2-O-acetyl aryl glycosides 1a-9a, the results are summarized in Table 1.

We found out that the monosaccharide moiety has a great influence on the regioselectivity of this reaction. Thus, for the compounds with the same aglycon regioselectivity (Table 1, column 4) decreases in the order: $\beta$-D-galactoside (3) > $\beta$-D-glucoside (2) >> $\alpha$-D-glucoside (9). In the case of glycoside 9 we detected several monoacetyl isomers and were not able to isolate individual products from this mixture. This fact indicates that 1,2-trans configuration of anomeric center is required for regioselective preparation of 2-O-acetyl glycosides.

In order to reveal the influence of aglycon on acetyl group reactivity we evaluated rate constants of acetyl group at O-2 of glycosides 1a-8a ethanolysis (Table 1, column 7). For this, 2O-acetyl glycosides 1a-8a were subjected to the reaction (as for 1a, Scheme 1, section 2.2) using the described conditions. We found out that the structure of the aglycon has a certain effect on the regioselectivity of the $\beta-\mathrm{D}$ aryl glycosides deacetylation. Thus, in the series of aryl glycoside 1 and para - substituted glycosides $4,6,8$, the regioselectivity decreases with the increasing of the electron-donating properties of the substituents. In addition, ortho - group in pairs of equal electronic substituents impedes the reaction and increases the yield of 2-O-acetyl product (entries $2,5,7$ in comparison to $4,6,8)$. 
It worth to note that in the case of methoxyphenyl glycosides $\mathbf{7}$ and $\mathbf{8}$ a substantial amount of isomeric monoacetates $7 \mathbf{c}$ and $8 \mathbf{c}$ were isolated (Table 1, entries 7 and 8, respectively). We established the structure of these isomers to be 3-O-acetyl glucopyranosides using NMR method. During the ethanolysis of glycosides 1-6 only small amount of isomeric monoacetates (presumably, 3-O-acetates) 1c-6c was detected by HPLC or not detected at all (were not isolated).

Table 1: Results of $\mathrm{HCl}$ - catalyzed deacetylation of per - acetylated glycosides 1-9

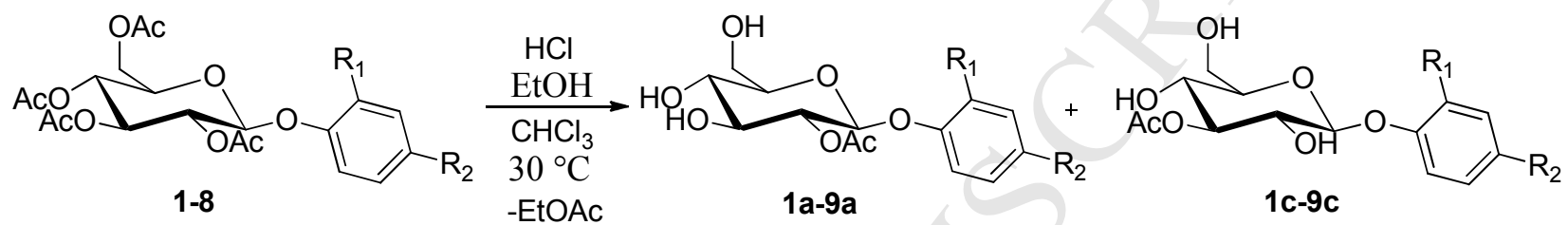

\begin{tabular}{|c|c|c|c|c|c|c|c|}
\hline $\begin{array}{l}\text { E } \\
\text { nt } \\
\text { ry }\end{array}$ & $\begin{array}{c}\text { Initial } \\
\text { compound/ } \\
\text { 2-O-acetyl } \\
\text { product }\end{array}$ & Substituent & $\begin{array}{c}\text { Retention } \\
\text { time of 2-O- } \\
\text { acetyl } \\
\text { product at } \\
\text { HPLC, min }\end{array}$ & $\begin{array}{c}\text { 2Ac: } 3 \mathrm{Ac} \\
\text { ratio }^{\mathrm{a}}\end{array}$ & $\begin{array}{l}\text { Reaction } \\
\text { time, } \mathrm{h}\end{array}$ & $\begin{array}{c}\text { Preparative } \\
\text { yield of 2- } \\
\text { O-acetyl } \\
\text { product, \% }\end{array}$ & Rate constant, ${ }^{\mathrm{b}} \mathrm{h}^{-1}$ \\
\hline & 1 & 2 & 3 & 4 & 5 & 6 & 7 \\
\hline 1 & 1/1a & $\mathrm{R}^{1}=\mathrm{R}^{2}=\mathrm{H}$ & 12.1 & $98: 2$ & 6 & 45 & $0.122 \pm 0.028$ \\
\hline 2 & $2 / 2 \mathbf{a}$ & $\mathrm{R}^{1}=\mathrm{CH}_{3}, \mathrm{R}^{2}=\mathrm{H}$ & 10.2 & $96: 4$ & 12 & 55 & $0.106 \pm 0.011$ \\
\hline 3 & $3 / 3 \mathbf{a}$ & $\begin{array}{c}\mathrm{R}^{1}=\mathrm{CH}_{3}, \mathrm{R}^{2}=\mathrm{H} \\
(\beta-\mathrm{D}-\text { Galp })^{\mathrm{c}}\end{array}$ & 10.2 & $100: 0$ & 11 & 35 & $0.084 \pm 0.008$ \\
\hline 4 & $4 / 4 a$ & $\mathrm{R}^{1}=\mathrm{H}, \mathrm{R}^{2}=\mathrm{CH}_{3}$ & 10.2 & $94: 6$ & 10 & 25 & $0.111 \pm 0.005$ \\
\hline 5 & 5/5a & $\mathrm{R}^{1}=\mathrm{NO}_{2}, \mathrm{R}^{2}=\mathrm{H}$ & 9.3 & 100:0 & 12 & 22 & $0.183 \pm 0.019$ \\
\hline 6 & 6/6a & $\mathrm{R}^{1}=\mathrm{H}, \mathrm{R}^{2}=\mathrm{NO}_{2}$ & 9.5 & $92: 8$ & 10 & 19 & $0.235 \pm 0.035$ \\
\hline 7 & $7 / 7 \mathbf{a}$ & $\mathrm{R}^{1}=\mathrm{OMe}, \mathrm{R}^{2}=\mathrm{H}$ & 8.8 & $85: 15$ & 12 & 45 & $0.282 \pm 0.034$ \\
\hline 8 & 8/8a & $\mathrm{R}^{1}=\mathrm{H}, \mathrm{R}^{2}=\mathrm{OMe}$ & 9.1 & $80: 20$ & 8 & 21 & $0.315 \pm 0.037$ \\
\hline 9 & 9/9a & $\begin{array}{c}\mathrm{R}^{1}=\mathrm{CH}_{3}, \mathrm{R}^{2}=\mathrm{H} \\
(\alpha-\mathrm{D}-\mathrm{Glcp})^{\mathrm{d}}\end{array}$ & 9.9 & $60: 30$ & $8^{\mathrm{e}}$ & - & ( \\
\hline
\end{tabular}

a) The ratio of glycoside 1a-9a to 1c-9c, correspondingly

b) Rate constants were determined for a single 2-O-acetyl group ethanolysis of glycosides

1a-8a to $\mathbf{1 b - 8 b}$ (see Scheme 1)

c) $\quad o$-cresyl $2,3,4,6$ tetra-O-acetyl- $\beta$-D-galactopyranoside was used as initial compound

d) $\quad o$-cresyl 2,3,4,6 tetra-O-acetyl- $\alpha$-D-glucopyranoside was used as initial compound

e) Time of maximal concentration of monoacetyl glycoside detected by HPLC

3.2 Mechanism modeling and the activation energy calculation 
The protonation step as the first step was modeled for each acetyl group of per - acetylated

glycoside 1. The geometries of each structure required for this step illustrating 2-O-acetyl group protonation are given in Figure 3. The calculated $\Delta \mathrm{Gs}$ of this step for each isomeric monoacetate (acetyl group at O-2, O-3, O-4 or O-6) are given in Table 2, the energy of reagents is taken for a zero. According to the results, the reaction is possible according to thermodynamic criteria at the temperature $\left(30{ }^{\circ} \mathrm{C}\right)$. The protonation of O-6 acetyl group carbonyl is the most energetically preferred because $\Delta \mathrm{Gs}$ for the protonation step of other acetyl groups (O-2, O-3 and O-4) are less for $7-10 \mathrm{kcal} / \mathrm{mol}$.

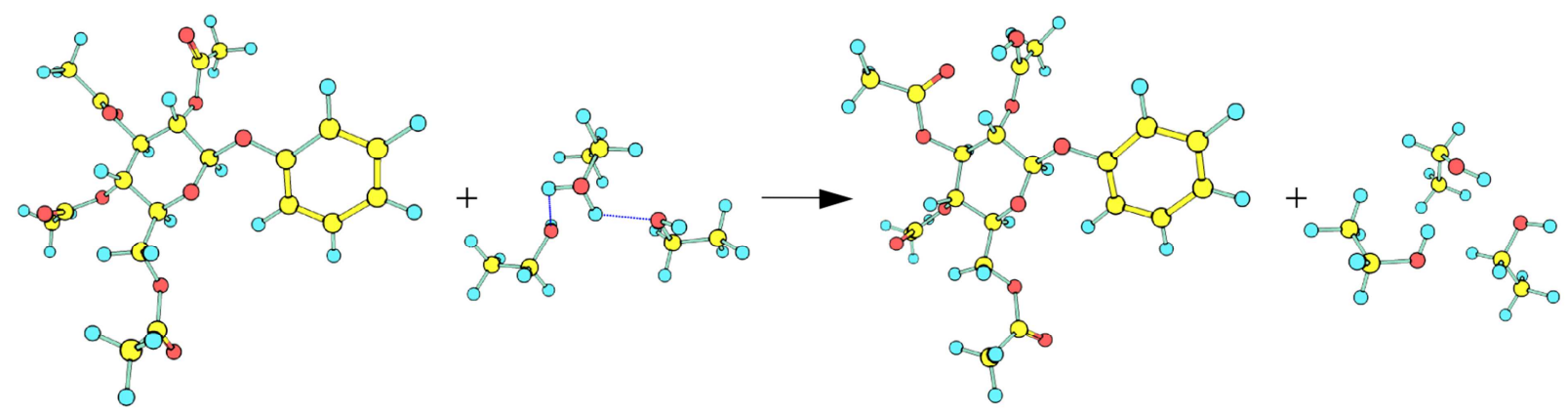

Figure 3: The modeling of protonation step of acid-catalysed deacetylation of compound 1 for protonation of 2-O-acetyl group

Table 2: Energies of products in the respect to reagents for the protonation step of acid-catalysed deacetylation of compound $\mathbf{1}$ calculated by AM1 method, in kcal/mol

\begin{tabular}{ccc}
\hline $\begin{array}{c}\text { Acetyl } \\
\text { group } \\
\text { protonated }\end{array}$ & $\begin{array}{c}\Delta \mathrm{G} \text { of the } \\
\text { protonation step }\end{array}$ & $\begin{array}{c}\text { Relative energies of } \\
\text { products }\end{array}$ \\
\hline O-2 & -132.4 & 10.2 \\
\hline O-3 & -132.4 & 10.2 \\
\hline O-4 & -135.0 & 7.6 \\
\hline O-6 & -142.6 & 0 \\
\hline
\end{tabular}

In general, the obtained results give thermodynamic characteristic of the first (protonation) step whereas do not explain the unusual reactivity of 2-O-acetyl group due to the second step (an interaction with the nucleophile) is rate-determining.

The optimized structures of RC, TS, PC for the second step (nucleophilic attack) of the reaction at O-2 of glycoside $\mathbf{1}$ are shown in Figure 4. The quantitative characteristics of this step 
for all isomers ( $\triangle \mathrm{Gs}$ between $\mathbf{R C}$ and $\mathbf{P C}$ and activation energies) are summarized in Table 3. As seen from Figure 4 (B), the imaginary frequency of TS involves the vibration of the proton which transfers from ethanol oxygen to glucose oxygen $\mathrm{O}-2$.
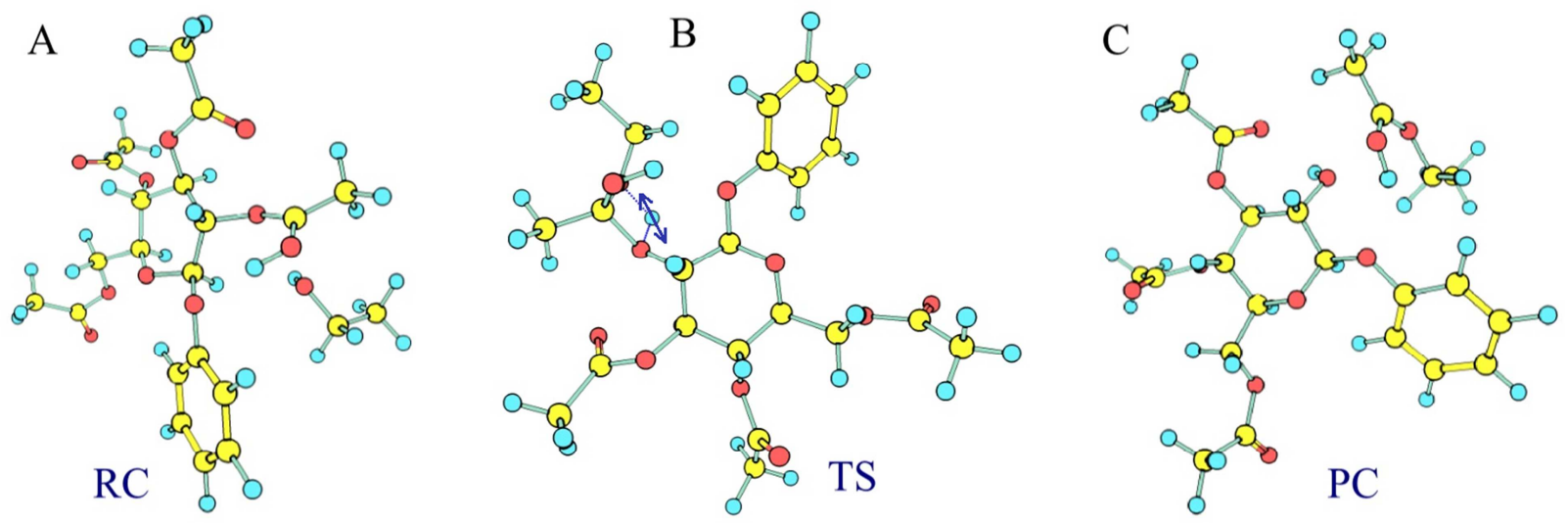

Figure 4: Optimized geometries for (A) RC, (B) TS (the imaginary frequency is illustrated by an arrow) and (C) PC of the ethanolysis reaction of O-2 acetyl group of per-acetylated phenyl glycoside 1.

Table 3: Relative energies of $\mathbf{R C}$, TS and in respect to the energy of PC; activation energies and Gibbs energies of the rate-determine stage of etanolysis of each acetyl group in tetrracetyl phenylglycoside 1 calculated by AM1 method, in $\mathrm{kcal} / \mathrm{mol}$

\begin{tabular}{cccccc}
\hline $\begin{array}{c}\text { Acetyl group } \\
\text { position }\end{array}$ & RC & TS & Activation energy & PC & $\Delta \mathrm{G}$ \\
\hline O-2 & 4.1 & 46.0 & 41.9 & 0 & -4.1 \\
\hline O-3 & 13.9 & 53.1 & 39.2 & 0 & -13.9 \\
\hline O-4 & 14.9 & 54.1 & 39.2 & 0 & -14.9 \\
\hline O-6 & 31.4 & 43.7 & 12.3 & 0 & -31.4 \\
\hline
\end{tabular}

According to the results all reactions are thermodynamically permitted. However, the activation barrier is maximal for the reaction at 2-O-acetyl group. Thus, it fully indicates the least reactivity of 2-O-acetyl group in acid-catalyzed reaction.

Assuming acetyl groups at 2, 3 and 4 positions react at similar concentrations the ratio $t=\exp \left(-E_{3,4} / k T\right) / \exp \left(-E_{2} / k T\right)$ gives a value of 88 , where $E_{i}$ is the activation energy of i-acetyl group. This rough estimation leads to conclusion that 3- and 4-O acetyl groups react almost two 
orders of magnitude faster than 2-O-acetyl group. 6-O-Acetyl group reacts much faster than all other acetyl groups because of activation barrier exceeds 3 times. However, the yields of 2-Oacetyl aryl glycosides in reaction of deacetylation are not high and not exceed $55 \%$. As noted above, the hydrolysis reaction may occur instead of ehanolysis. Therefore, the interaction between water molecule and the protonated glycoside $\mathbf{1}$ can be involved in the step of transition stay formation (Table 4 shows this interaction for 2-O-acetyl group). The results of activation energy calculation of transition states (TS) of hydrolysis are summarized in Table 4. Although concentration of water is very negligible in comparison with ethanol as solvent, this reaction can reduce the yield of 2-O acetyl glycosides due to the activation barrier is almost same and very small for all acetyl groups.

Table 4 - Activation energies of the rate-determine stage of hydrolysis of each acetyl group of tetraacetyl phenylglycoside 1 calculated by AM1 method, in $\mathrm{kcal} / \mathrm{mol}$

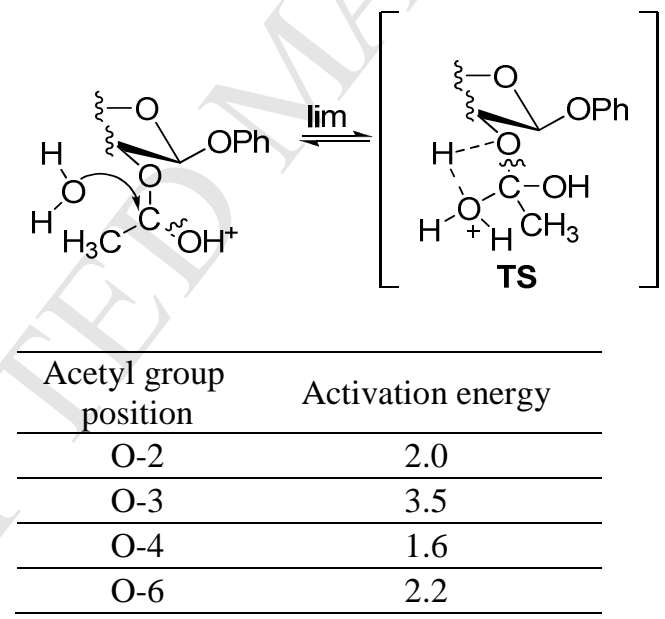

Also, we calculated the activation barrier for 2-O-acetyl group of 1a deacetylation. The results of quantum-chemical calculations using both AM1 and DFT/B3LYP methods as well as experimental data are summarized in Table 5. According to the results, AM1 method leads to an insignificant overestimation of activation energy by $9 \mathrm{kcal} / \mathrm{mol}$ and generally gives an adequate values, therefore, we can assume that the above calculated activation energies using the AM1 method are acceptable for the reactivity evaluation. The results obtained using DFT/B3LYP method are in a good agreement with the experiment. Thus, activation energies given in Tables 2 
and 3 can be overestimated by $9 \mathrm{kcal} / \mathrm{mol}$ in general; nevertheless, the calculated results correctly reproduce the regularity of acetyl groups of per - acetylated glycoside reactivity in acid-catalyzed deacetylation. Also, the together experimental and theoretical results show that the ethanolysis mechanism prevails over hydrolysis in spite of small activation barrier for the latter.

Table 5: Activation energy for the reaction of 2-O-acetyl group ethanolysis of 2-O-acetyl phenyl glucopyranoside 1a

\begin{tabular}{cc} 
Calculation method & $\begin{array}{l}\text { Activation energy, } \\
\text { in kcal/mol }\end{array}$ \\
\hline $\begin{array}{c}\text { DFT/B3LYP/6- } \\
\text { 31G(d,p) }\end{array}$ & 25.3 \\
\hline AM1 & 30 \\
\hline ExP & 21.4
\end{tabular}

\section{Conclusions}

Thus, we established that the reactivity of 2-O-acetyl group of per - acetylated $\beta$-D-aryl glycosides (glucopyranosides and galactopyranosides) is less than other acetyl groups during the ethanolysis reaction using $\mathrm{HCl} / \mathrm{EtOH}$ in $\mathrm{CHCl}_{3}$. This result gives the base for the synthetic method for the preparation of 2-O-acetyl aryl glycosides. The least reactivity of 2-O-acetyl group is explained by the highest activation barrier of rate-determining step of nucleophilic attack for the formation of tetrahedral transition state according to the quantum-chemical calculations. According to our estimation, the reactivity of 2-O-acetyl group is 88 times less than for 3-, 4- or 6-acetyl groups.

\section{Supplementary material}

Spectral data, physicochemical properties of monoacetyl glycosides $\mathbf{1 a - 8 a , 7 c}$ and $\mathbf{8 c}$ as well as ${ }^{1} \mathrm{H},{ }^{13} \mathrm{C}$, and 2D NMR spectra are given in the Support information file $\mathbf{1}$. The geometries of calculated molecules (RC, PC) and transition states (TS) are given in the Support information 2.

\section{Acknowledgements}


This work was supported by the Tomsk Polytechnic University Competitiveness

Enhancement Program grant [Project Number VIU-TOVPM-316/2017 and by The Tomsk State

University Competitiveness Improvement Programme grant (N8.1.03.2017).

\section{References}

[1] G.A. Boeckler, J. Gershenzon, S.B. Unsicker, Phytochem. 72 (2011) 1497-1509.

[2] W.M. Abdel-Mageed, E.Y. Backheet, A.A. Khalifa, Z.Z. Ibraheim, S.A. Ross, Fitoterapia. 83 (2012) 500-507.

[3] V. Zsoldos-Mády, A. Csámpai, R. Szabó, E. Mészáros-Alapi, J. Pásztor, F. Hudecz, P. Sohár, Chem. Med. Chem. 1 (2006) 1119-1125.

[4] I.N. Abreu, M. Ahnlund, T. Moritz, B. R. Albrectsen, J. Chem. Ecol. 37 (2011) 857870.

[5] K. Keefover-Ring, M. Ahnlund, I.N. Abreu, S. Jansson, T. Moritz, B.R. Albrectsen PloS one 9 (2014) e107189.

[6] E. Dagvadorj, K.H. Shakeret, D.Windsor, B.Schneider, W. Boland, Phytochem. 71 (2010) 1900-1907.

[7] P.B. Reichardt, H.M. Merken, T.P. Clausen, J. Wu, J. Nat. Prod. 55 (1992) 970-973.

[8] M. Lee, S.H. Lee, J. Kang, H. Yang, E.J. Jeong, H.P. Kim, Y.C. Kim; S.H. Sung, Molecules. 18 (2013) 10484-10496.

[9] C.S. Kim, L. Subedi, K.J. Park, S.Y. Kim, S.U. Choi, K.H. Kim, K.R. Lee, Fitoterapia. 106 (2015) 147-152.

[10] M.U. Roslund, O. Aitio, J. Warna, H. Maaheimo, D.Yu. Murzin, R. Leino, J. Am. Chem. Soc. 130 (2008) 8769-8772.

[11] L. Brecker, M. Mahut, A. Schwarz, B. Nidetzky, Magn. Reson. Chem. 47 (2009) 328332.

[12] M. Mastihubová, P. Biely, Carbohydr Res, 339 (2004) 1353-1360.

[13] D. Horton, W.E. Mast, K.D. Philips, J. Org. Chem. 32 (1967), 1471-1474.

[14] R.L. Lehtila, J.O. Lehtila, M.U. Roslund, R. Leino, Tetrahedron. 60 (2004) 36533661 .

[15] T.W.Greene, P.G.M. Wuts, Protective Groups in Organic Synthesis; John Wiley and Sons Inc.: New York, 2007.

[16] J. Sun, X. Han, B. Yu, Synlett. 3 (2005) 437- 440.

[17] Zemplén Deacetylation. Comprehensive Organic Name Reactions and Reagents. 691 (2010) 3123-3128. 
[18] U. Ellervik, G. Magnusson, Tetrahedron Let. 38 (1997) 1627-1628.

[19] B. Ren, M. Wang, J. Liu, J. Ge, X. Zhang, H. Dong, Green Chem. 17 (2015) 13901394.

[20] D. Artner, C. Stanetty, K. Mereiter, A. Zamyatina, P. Kosma, Carbohydr. Res. 346 (2011) 1739-1746.

[21] N.É. Byramova, M.V. Ovchinnikov, L.V. Backinowsky, N.K. Kochetkov, Carbohydr. Res. 124 (1983) C8-C11.

[22] A.G. González, I. Brouard, F. León, J.I. Padrón, J. Bermejo, Tetrahedron Lett. 42 (2001) 3187-3188.

[23] C.E. Yeom, S.Y. Lee, Y.J. Kim, B.M. Kim, Synlett, 10 (2005) 1527-1530.

[24] E.V. Stepanova, M.O. Nagornaya, M.L. Belyanin, V.D. Filimonov, Curr. Org. Synth. 14 (2017) 394-397.

[25] E.V. Stepanova, M.L. Belyanin, V.D. Filimonov, Carbohydr. Res. 388 (2014) 105111.

[26] A. Cabiac, E. Guillon, F. Chambon, C. Pinel, F. Rataboul, N. Essayem, Appl. Catal. A: Gen. 402 (2011) 1-10.

[27] E.V. Stepanova, M.L. Belyanin, V.D. Filimonov, R.R.Valiev, M. Gruner, V. Rogachev, Carbohydr. Res. 409 (2015) 36-40.

[28] Y.S. Lee, E.S. Rho, Y.K. Min, B.T. Kim, K.H. Kim, Carbohydr. Chem. 20 (2001) 503-506.

[29] J.N.E. Day, C.K. Ingold, Trans. Faraday Soc. 37 (1941) 686-705.

[30] L. Reyes, I. Nicolas-Vazquez, N. Mora-Diez, J.R. Alvarez-Idaboy, J. Org. Chem. 78 (2013) 2327-2335.

[31] M.J.S. Dewar, E.G. Zoebisch, E.F. Healy, J. Am. Chem. Soc. 107 (1985) 3902-3909.

[32] R.O. Jones, Rev. Mod. Phys. 87 (2015) 897-923.

[33] A. D. Becke, J. Chem. Phys. 98 (1993) 5648-5652.

[34] N.B. Librovich, E.G. Tarakanova, Dokl. Akad. Nauk. 410 (2006) 357-360.

[35] S. Miertuš, E. Scrocco, J. Tomasi, Chem. Phys. 55 (1981) 117-29.

[36] A. Klamt, G. Schüürmann, J. Chem. Soc., Perkin Trans. 2. 0 (1993) 799-805.

[37] M. J. Dewar, C. Jie, E.G. Zoebisch, Organometallics. 7 (1988) 513-521.

[38] S.J. Branko, J. Mol. Struct. THEOCHEM, 358 (1995) 139-143. 
[39] Frisch M, Trucks G, Schlegel H., Scuseria G., Robb M, Cheeseman J, Scalmani G.,

Barone V, Mennucci B, Petersson G, Nakatsuji H, Caricato M, Li X, Hratchian H, Izmaylov A, Bloino J, Zheng G, Sonnenberg J, Hada M, Ehara M, Toyota K, Fukuda R, Hasegawa J, Ishida M, Nakajima T, Honda Y, Kitao O, Nakai H, Vreven T, Montgomery J, Jr, Peralta J, Ogliaro F, Bearpark M, Heyd J, Brothers E, Kudin K, Staroverov V, Kobayashi R, Normand J, Raghavachari K, Rendell A, Burant J, Iyengar S, Tomasi J, Cossi M, Rega N, Millam J, Klene M, Knox J, Cross J, Bakken V, Adamo C, Jaramillo J, Gomperts R, Stratmann R, Yazyev O, Austin A, Cammi R, Pomelli C, Ochterski J, Martin R, Morokuma K, Zakrzewski V, Voth G, Salvador P, Dannenberg J, Dapprich S, Daniels A, Farkas Ö, Foresman J, Ortiz J, Cioslowski J, Fox D (2009) Gaussian 09, revision A.02. Gaussian, Inc., Wallingford CT. 
Highlights:

1. We revealed different reactivity of acetyl groups of glycosides in acid-catalyzed deacetylation

2. Regioselective preparation of 2-O-acetyl aryl glycosides was accomplished

3. The influence of carbohydrate structure on regioselectivity was revealed

4. Different reactivity of Ac groups is explained by activation barriers 